\title{
Effect of dual antiplatelet on recurrent stroke in minor stroke or TIA depends on bodyweight
}

This article was published in the following Dove Press journal:

Therapeutics and Clinical Risk Management

\author{
Yan $\mathrm{Ma}^{\mathrm{I}}$ \\ Ying Liu ${ }^{2-5}$ \\ Jie $\mathrm{Xu}^{2-5}$ \\ Yilong Wang ${ }^{2-5}$ \\ Yongjun Wang ${ }^{2-5}$ \\ Fenghe $\mathrm{Du}^{\prime}$ \\ 'Department of Cardiology, Beijing \\ Tiantan Hospital, Capital Medical \\ University, Beijing, China; ${ }^{2}$ Department \\ of Neurology, Beijing Tiantan Hospital, \\ Capital Medical University, Beijing, \\ China; ${ }^{3}$ China National Clinical \\ Research Center for Neurological \\ Diseases, Beijing, China; ${ }^{4}$ Center of \\ Stroke, Beijing Institute for Brain \\ Disorders, Beijing, China; ${ }^{5}$ Beijing Key \\ Laboratory of Translational Medicine \\ for Cerebrovascular Disease, \\ Beijing, China
}

Objective: To assess whether bodyweight influences the efficacy and safety of dual antiplatelet therapy (DAT) in male patients with minor stroke or transient ischemic attack patients.

Materials and methods: All 3,420 male participants coming from the Clopidogrel in HighRisk Patients with Acute Non-disabling Cerebrovascular Events trial were divided into 3 groups based on bodyweight ( $<65 \mathrm{~kg}, 65-75 \mathrm{~kg}$, and $\geq 75 \mathrm{~kg}$ ). The stroke outcomes included stroke recurrence, combined vascular events, and bleeding events during 90 days of follow-up. The interaction of the treatment effects of DAT among patients with different bodyweight was assessed by Cox proportional hazards models.

Results: DAT is superior to mono antiplatelet therapy (MAT) for reducing stroke recurrence among patients with weight $<65 \mathrm{~kg}(5.0 \%$ vs $11.7 \%$; hazard ratio [HR], 0.41 ; 95\% CI: $0.22-0.76)$ and $65-75 \mathrm{~kg}(6.7 \%$ vs $10.8 \%$, HR, 0.62; 95\% CI: 0.43-0.89). However, no significant difference was found in stroke recurrence between DAT and MAT in patients with weight $\geq 75 \mathrm{~kg}$ (9.4\% vs $11.6 \%$; HR, 0.80; 95\% CI: 0.58-1.10). A significant interaction was observed between weight and antiplatelet therapy on stroke recurrence $(p<0.05)$. Similar result was found for combined vascular events. More bleeding events were found in DAT group among patients with weight $<65 \mathrm{~kg}$ (3.7\% vs $2.2 \%)$, but with no significant difference.

Conclusion: DAT does not show benefit in patients with higher weight, compared with MAT. Bleeding events found in the DAT group were not more than the MAT group among patients with lower weight.

Clinical trial registration: URL: http://www.ClinicalTrials.gov. Unique identifier: NCT00979589.

Keywords: bodyweight, dual antiplatelet therapy, ischemic stroke, outcomes, TIA

\section{Introduction}

Dual antiplatelet therapy (DAT) is an effective way in reducing the risk of early stroke recurrence and other vascular events as compared with mono antiplatelet therapy (MAT) in patients with acute ischemic stroke or transient ischemic attack (TIA). ${ }^{1-3}$ After Clopidogrel in High-Risk Patients with Acute Non-disabling Cerebrovascular Events (CHANCE) study reported that DAT was superior to MAT for reducing the risk of stroke in the first 90 days, the American Heart Association/American Stroke Association recommended that the combination of aspirin and clopidogrel can be initiated within $24 \mathrm{~h}$ for a minor ischemic stroke or TIA and be continued for 90 days in 2014. ${ }^{4}$ It is not clear which subgroup can benefit from the DAT. ${ }^{5}$ We can find an interesting phenomenon that DAT seemed superior to MAT in Eastern population but not in Western. ${ }^{1,3,6,7}$ This discrepancy might be attributed to the different demographic characteristics between 2 populations, bodyweight was an obvious disparity. ${ }^{8}$ Some studies showed that higher weight was associated with more vascular recurrence 
after stroke. ${ }^{9}$ Whether bodyweight may influence the effect of antiplatelet therapy on stroke recurrence in patients with ischemic stroke or TIA is unreported. We sought to assess whether bodyweight would influence the efficacy and safety of DAT in minor stroke and TIA patients in the cohort of the CHANCE trial.

\section{Materials and methods Data sources}

We used data from CHANCE trial. Details on the rationale, design, and early results have been published previously.,10 The study was approved by medical ethics committee of Beijing Tiantan Hospital. The CHANCE trial was a randomized, double-blind, placebo-controlled clinical trial conducted at 114 centers in China between October 2009 and July 2012. The complete list of investigators and institutions participating in the CHANCE trial is provided in the Supplementary material. Patients were recruited if they met the following criteria: $\geq 40$ years of age, had a diagnosis of an acute minor stroke or high-risk TIA, and were able to start the study drug within $24 \mathrm{~h}$ after onset of symptom. All eligible patients were randomly assigned to the following therapy arms: a placebo version of Plavix (clopidogrel bisulfate; Sanofi Pharmaceuticals Partnership, Bridgewater, NJ, USA) on days 1 through 90, plus aspirin at a dose of $75 \mathrm{mg}$ per day on days 2 through 90; or $300 \mathrm{mg}$ of clopidogrel on day 1 , followed by a dose of $75 \mathrm{mg}$ per day on days 2 through 90, plus Aspirin (Friedrich Bayer \& Co., Elberfeld, Germany) at a dose of $75 \mathrm{mg}$ per day on days 2 through 21, and placebo aspirin on days 22 through 90. Acute minor stroke was defined by a score of 3 or less on the National Institutes of Health Stroke Scale (NIHSS) upon randomization. TIA was defined as focal brain ischemia with resolution of symptoms within $24 \mathrm{~h}$ after onset, and with a score of $\geq 4$ on the ABCD2 when randomized. Study visits were planned on the day of randomization, 21, and 90 days after randomization and at hospital discharge. Details of the information about patients' clinical status, interim occurrence of any adverse events, and medication compliance were collected.

\section{Study population}

Bodyweight is a gender-specific variable. Gender-related differences in pharmacokinetics have frequently been considered as potentially important determinants for the clinical effectiveness of drug therapy. ${ }^{11}$ Thus, in this subgroup we only investigate the effect of bodyweight on DAT in one gender. Males were a majority in the CHANCE trial. Bigger sample size can give us greater power to detect differences, so we analyzed 3,420 male patients in this subgroup.

\section{Bodyweight measurement and classification}

The bodyweight was measured by the study coordinator at the initial randomization visit. Each participant was weighed in lightweight clothing, with the measurement taken on a calibrated beam scale, and the weight recorded to the nearest $1 \mathrm{~kg}$. Average weight of an adult male in China was $66.2 \mathrm{~kg}$ in 2012 according to a Chinese government report. ${ }^{12}$ We classified bodyweight as low bodyweight (weight $<65 \mathrm{~kg}$ ), middle bodyweight ( $65 \mathrm{~kg} \leq$ weight $<75 \mathrm{~kg}$ ), and high bodyweight (weight $\geq 75 \mathrm{~kg}$ ).

\section{Efficacy and safety outcome}

Written informed consent was obtained from all participants or their legal proxies. CHANCE protocol was approved by the ethics committee at each study center. The primary efficacy outcome was recurrent stroke (ischemic or hemorrhagic) within 90 days. ${ }^{1}$ The secondary efficacy outcome included combined vascular events (CVEs) (ischemic stroke, hemorrhagic stroke, myocardial infarction, or vascular death). The primary safety outcome was any bleeding event, as per the Global Utilization of Streptokinase and Tissue Plasminogen Activator for Occluded Coronary Arteries (GUSTO) definition. ${ }^{13}$ All reported efficacy and safety outcomes were confirmed by a central adjudication committee that was blinded to the study group assignments.

\section{Statistical analysis}

Continuous and categorical variables were reported as mean \pm SD or percentages and compared by use of Student's $t$-test, Fisher's exact test or $\chi^{2}$ test ( 2 tailed), respectively. Furthermore, we performed survival analysis and present Kaplan-Meier curves by different groups of bodyweight for stroke and CVE. Multivariate Cox regressions were performed to assess the associations between bodyweight and clinical outcome variables. Interaction effect between bodyweight and antiplatelet was also tested in Cox model.

The adjusted variables, including age, gender, current or previous smoking, current or previous drinking, history of ischemic stroke or TIA, myocardial infarction, hypertension and hyperlipidemia, final diagnosis of an acute minor ischemic stroke or TIA, the use of lipid-lowering agents and antihypertension agents during 90-day follow-up period. All $p$-values were 2 -sided, with values of $p<0.05$ considered 
statistically significant. All statistical analyses were performed using SAS Version 9.4 software (SAS Institute, Cary, NC, USA). As this was a post hoc analysis, we considered these analyses to be hypothesis generating.

\section{Search terms}

[12] Stroke prevention; [13] Other cerebrovascular disease/ stroke.

\section{Results}

\section{Patient demographics and baseline characteristics}

Among the 5,170 patients recruited in CHANCE, all the 3,420 (66.2\%) male patients were included in this subgroup analysis. Participant demographics and characteristics are presented in Table 1. Patients with higher bodyweight were younger, more likely to be current or previous smokers and drinkers. They tended to have a history of TIA or ischemic stroke, myocardial infarction, hypertension, diabetes mellitus, and hypercholesterolemia, and most of them had TIA as a qualifying event. In addition, the rates of using antihypertensive at baseline were higher in patients included in the high-weight group, but the rates of using lipid-loweringmedicine were highest in the low-weight group. In each group, no significant difference was found with previous baseline information between DAT and MAT patients.

\section{Efficacy outcomes}

Overall, 320 patients (9.4\%) in the current subgroup analysis had a primary efficacy outcome of recurrent stroke at 90 days. The relative proportion of patients with stroke recurrence was different between DAT and MAT in low bodyweight group ( $p=0.003$ ) and middle bodyweight group ( $p=0.007)$, but no significant difference was found in high bodyweight group ( $p=0.172$ ) (Figure 1). A similar result was found for CVE. In low and middle bodyweight groups, the risks with CVE in DAT and MAT were different ( $p=0.003$ and $p=0.007$, respectively) but not in high bodyweight group $(p=0.173)$ (Figure 2). As shown in Figure 3, significant interactions were found between bodyweight and randomized antiplatelet therapy in their effects on both stroke and CVE in low and middle bodyweight groups. In patients with low and middle bodyweight, combination therapy of clopidogrel and aspirin obviously reduced the occurrence of stroke and CVE compared with aspirin alone. The crude hazard ratio (HR) for clopidogrel plus aspirin vs aspirin alone on the primary outcome of any stroke in patients with low bodyweight was 0.41 (95\% CI: 0.22-0.76) and the adjusted HR 0.41 (95\% CI: $0.22-0.76)$. As in middle bodyweight group, the crude HR of total stroke for clopidogrel plus aspirin vs aspirin alone in patients with low bodyweight was 0.61 (95\% CI: 0.42-0.88) and the adjusted HR 0.41 (95\% CI: 0.43-0.89). However, in the patients with high bodyweight, no difference was found

Table I Demographic and clinical characteristics of patients according to the bodyweight in the current subgroup analysis of the CHANCE trial

\begin{tabular}{|c|c|c|c|c|c|c|c|c|c|}
\hline \multirow[t]{2}{*}{ Variables } & \multicolumn{2}{|c|}{ Weight $<65$ kg } & \multirow[t]{2}{*}{$p$-value } & \multicolumn{2}{|c|}{$65 \mathrm{~kg} \leq$ weight $<75 \mathrm{~kg}$} & \multirow[t]{2}{*}{$p$-value } & \multicolumn{2}{|c|}{ Weight $\geq 75$ kg } & \multirow[t]{2}{*}{$p$-value } \\
\hline & $\begin{array}{l}\text { Clopidogrel- } \\
\text { aspirin } \\
(n=301)\end{array}$ & $\begin{array}{l}\text { Aspirin } \\
(n=274)\end{array}$ & & $\begin{array}{l}\text { Clopidogrel- } \\
\text { aspirin } \\
(n=706)\end{array}$ & $\begin{array}{l}\text { Aspirin } \\
(n=688)\end{array}$ & & $\begin{array}{l}\text { Clopidogrel- } \\
\text { aspirin } \\
(n=725)\end{array}$ & $\begin{array}{l}\text { Aspirin } \\
(n=726)\end{array}$ & \\
\hline $\begin{array}{l}\text { Age (years), } \\
\text { mean } \pm \text { SD }\end{array}$ & $65.9 \pm 10.8$ & $64.6 \pm 10.8$ & 0.14 & $62.0 \pm 10.5$ & $61.9 \pm 10.4$ & 0.87 & $60.3 \pm 10.8$ & $59.6 \pm 10.8$ & 0.21 \\
\hline \multicolumn{10}{|c|}{ Medical history-no. (\%) } \\
\hline TIA or ischemic stroke & $61(20.3)$ & $60(21.9)$ & 0.63 & $169(23.9)$ & $164(23.8)$ & 0.96 & $204(28.1)$ & 177 (24.4) & 0.1 \\
\hline Myocardial infarction & $8(2.7)$ & $3(52.2)$ & 0.17 & $14(2.0)$ & $20(2.9)$ & 0.26 & 14 (I.9) & $18(2.5)$ & 0.48 \\
\hline Hypertension & $164(54.5)$ & 143 (52.2) & 0.58 & $433(61.3)$ & $417(60.6)$ & 0.78 & $517(71.3)$ & $501(69.0)$ & 0.34 \\
\hline Diabetes mellitus & $40(13.3)$ & $39(14.2)$ & 0.74 & $108(15.3)$ & 133 (19.3) & 0.05 & $166(22.9)$ & 158 (2I.8) & 0.6 \\
\hline Hypercholesterolemia & $18(6.0)$ & $22(8.0)$ & 0.33 & $69(9.8)$ & $71(10.3)$ & 0.73 & $105(14.5)$ & $94(13.0)$ & 0.4 \\
\hline $\begin{array}{l}\text { Current or previous } \\
\text { smoking-no. (\%) }\end{array}$ & $183(60.8)$ & $163(59.5)$ & 0.75 & $433(6 \mid .3)$ & $413(60.0)$ & 0.62 & $459(63.3)$ & $47 I$ (64.9) & 0.53 \\
\hline $\begin{array}{l}\text { Current or previous } \\
\text { drinking-no. (\%) }\end{array}$ & $128(42.5)$ & I I 8 (43.I) & 0.9 & $312(44.2)$ & 302 (43.9) & 0.91 & $356(49.1)$ & $356(49.0)$ & 0.98 \\
\hline \multicolumn{10}{|c|}{ Qualifying event-no. (\%) } \\
\hline TIA & $74(24.6)$ & $68(24.8)$ & 0.95 & $188(26.6)$ & $182(26.5)$ & 0.94 & $209(28.8)$ & $203(28.0)$ & $0.7 \mathrm{I}$ \\
\hline Minor stroke & $227(75.4)$ & $206(75.2)$ & & $518(73.4)$ & $506(73.5)$ & & $516(7 I .2)$ & $523(72.0)$ & \\
\hline \multicolumn{10}{|c|}{ Secondary prevention-no./total (\%) } \\
\hline Anti-hypertension & $\begin{array}{l}91 / 277 \\
(30.6)\end{array}$ & $\begin{array}{l}87 / 271 \\
(32.1)\end{array}$ & $0.7 \mathrm{I}$ & $\begin{array}{l}247 / 701 \\
(35.2)\end{array}$ & $\begin{array}{l}217 / 681 \\
(31.9)\end{array}$ & 0.18 & $\begin{array}{l}258 / 725 \\
(35.8)\end{array}$ & $\begin{array}{l}256 / 720 \\
(35.3)\end{array}$ & 0.84 \\
\hline Lowering-lipid & $\begin{array}{l}132 / 277 \\
(44.4)\end{array}$ & $\begin{array}{l}|2| / 27 \mid \\
(44.7)\end{array}$ & 0.96 & $\begin{array}{l}310 / 701 \\
(44.2)\end{array}$ & $\begin{array}{l}274 / 681 \\
(40.2)\end{array}$ & 0.13 & $\begin{array}{l}307 / 725 \\
(42.6)\end{array}$ & $\begin{array}{l}315 / 720 \\
(43.5)\end{array}$ & 0.76 \\
\hline
\end{tabular}

Abbreviations: CHANCE, Clopidogrel in High-Risk Patients with Acute Non-disabling Cerebrovascular Events; TIA, transient ischemic attack. 

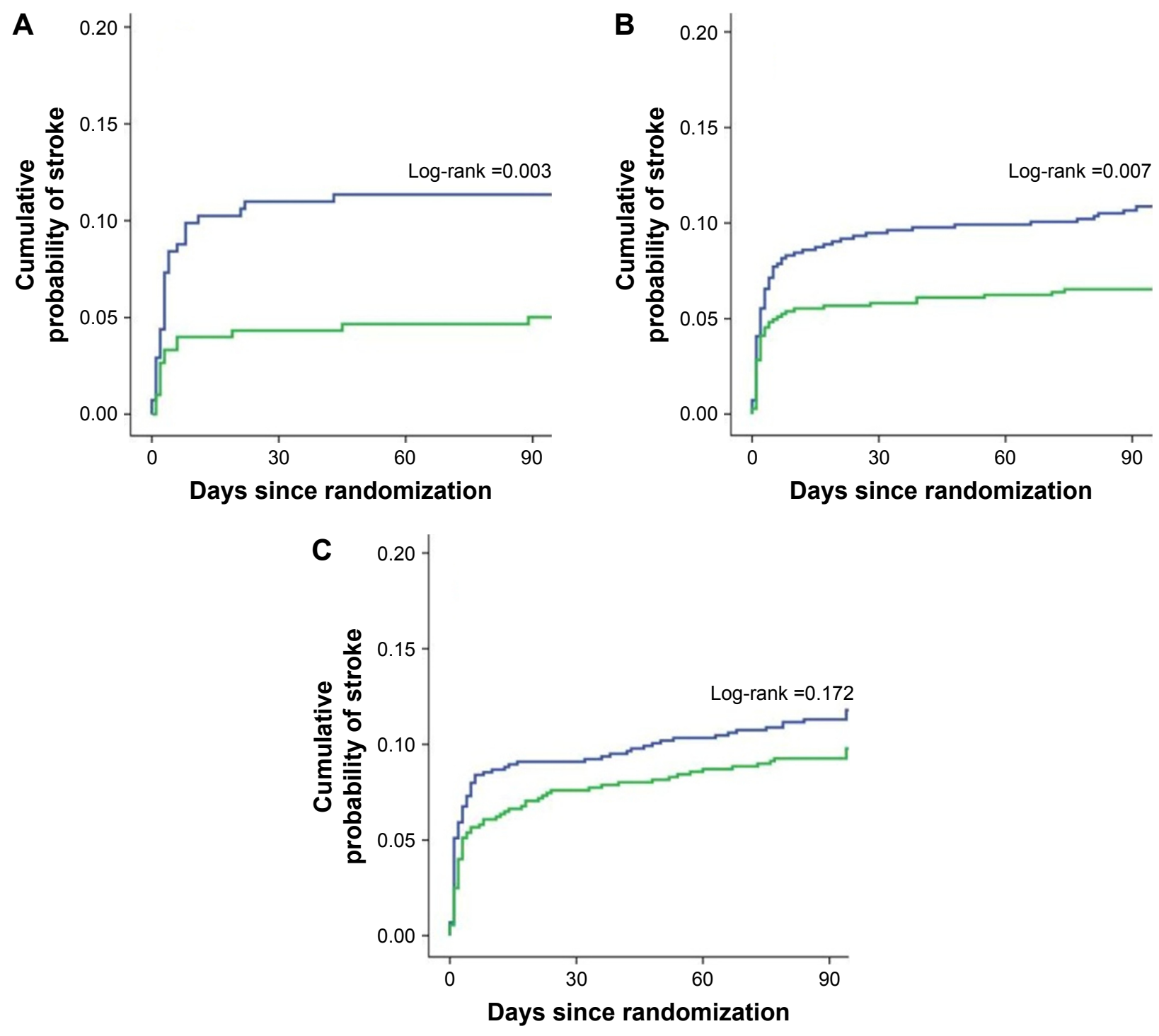

$\neg$ Aspirin $\backsim$ Clopidogrel-aspirin

Figure I Kaplan-Meier curves for the primary efficacy outcome of any stroke.

Notes: Kaplan-Meier curves showing the time to the primary efficacy outcome event (any stroke) in patients with different bodyweight, treated with placebo plus aspirin, or clopidogrel plus aspirin. (A) Low bodyweight (weight $<65 \mathrm{~kg}$ ); (B) middle bodyweight (65 kg $\leq$ weight $<75 \mathrm{~kg}$ ); (C) high bodyweight (weight $\geq 75 \mathrm{~kg}$ ).

in the rate of stroke or CVE between DAT and MAT. The crude HR of stroke was 0.80 (95\% CI: $0.58-1.10)$ and the adjusted HR 0.80 (95\% CI: 0.58-1.10).

\section{Safety outcomes}

The rates of the primary safety outcome of moderate-tosevere bleeding events at 90 days were both extremely low in the patients of 3 different weight groups as shown in Table 2. Moderate or severe hemorrhage, as defined by means of the GUSTO criteria, occurred in 2 patients with DAT and 1 patient with MAT in low bodyweight group. The rate of any bleeding event was $3.7 \%$ with DAT and $2.2 \%$ with MAT in low bodyweight group. No statistically significant evidence was found for the interaction on the effect of clopidogrel plus aspirin vs aspirin alone on bleeding events among patients with different bodyweight (Figure 3).

\section{Discussion}

In this subgroup analysis of the CHANCE trial, a better therapeutic response with DAT than MAT was found in male patients with low and middle bodyweight. Compared with MAT, DAT did not show superior benefit in high bodyweight patients. A significant interaction of bodyweight with randomized antiplatelet therapy was observed. DAT was not associated with an increase in incidence of bleeding events in all 3 bodyweight groups. 

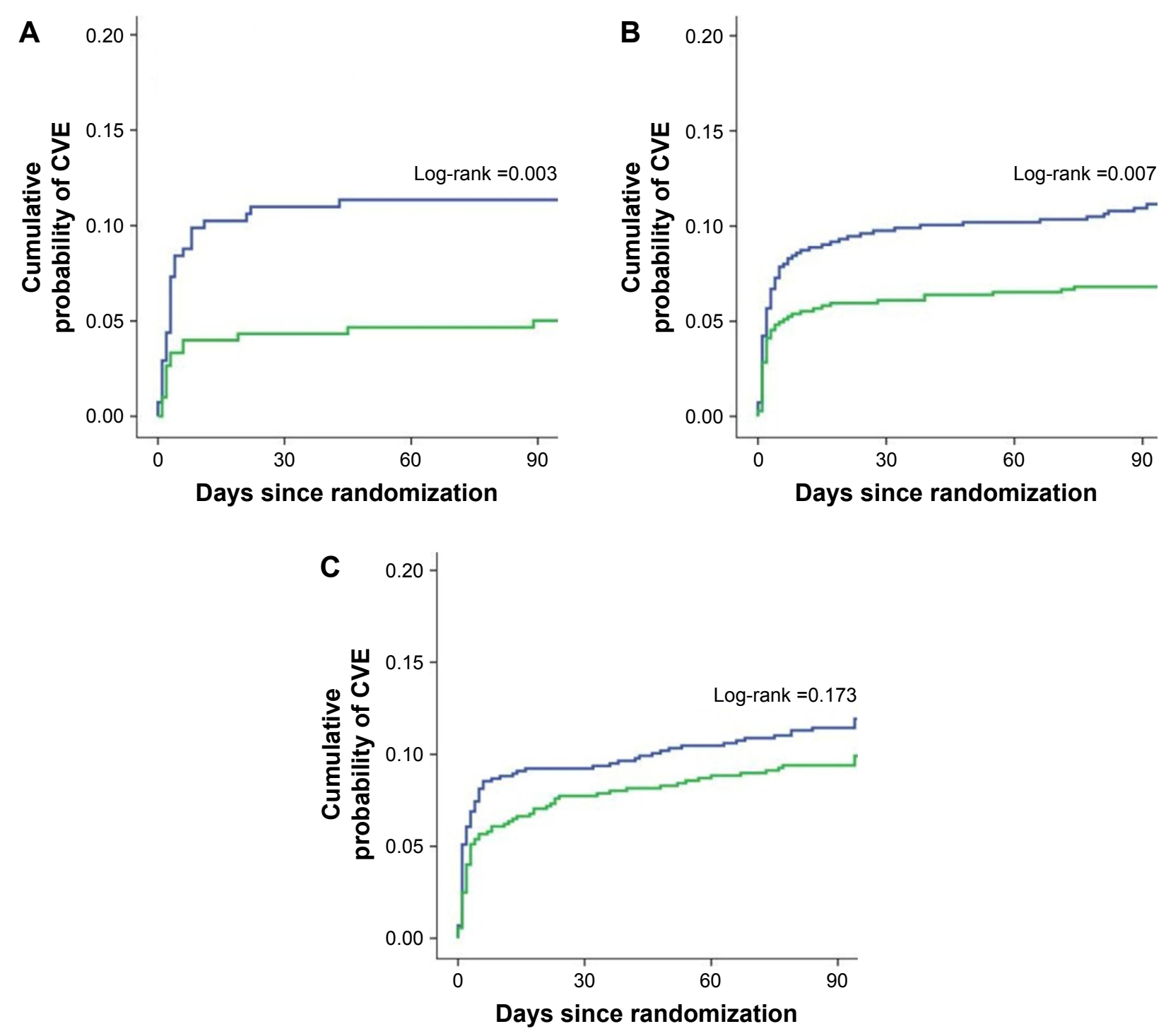

$\neg$ Aspirin $\backsim$ Clopidogrel-aspirin

Figure 2 Kaplan-Meier curves for the secondary efficacy outcome of CVE.

Notes: Kaplan-Meier curves showing the time to the secondary efficacy outcome event (CVE) in patients with different bodyweight treated with placebo plus aspirin, or clopidogrel plus aspirin. (A) Low bodyweight (weight $<65 \mathrm{~kg}$ ); (B) middle bodyweight (65 kg $\leq$ weight $<75 \mathrm{~kg}$ ); (C) high bodyweight (weight $\geq 75 \mathrm{~kg}$ ).

Abbreviation: CVE, combined vascular event.

DAT as the secondary prevention of stroke has been studied in many randomized controlled trials (RCTs). These RCTs were distinct from each other in design. Ongoing POINT trial is assessing a higher loading dose of clopidogrel $(600 \mathrm{mg})$ than CHANCE. ${ }^{14}$ The higher loading dose may be partly due to the bigger body size of the included population. The CHANCE and CLAIR trials which were conducted in Eastern populations preferred DAT, while CHARISMA and FASTER trials carried out in Western populations reported no advantage of DAT vs MAT. 1,3,6,7,14 No more benefits of DAT documented in Western population may be partly attributable to their higher bodyweight than Eastern populations. DAT using aspirin and clopidogrel is of great importance after acute non-disabling cerebrovascular events nowadays. But 1 antiplatelet therapy algorithm does not fit all. To identify patients with the risk of inadequate DAT is of significant clinical practice. Recently, as obesity and overweight are increasing, more and more researchers show interest in the effect of bodyweight on the effectiveness of antiplatelet therapy. ${ }^{15-19}$

The mechanisms responsible for the no superior effect with DAT in the high bodyweight population may be elucidated as follows. First, DAT is still not sufficient to prevent stroke events in heavier patients. So one underlying explanation for no difference between DAT vs MAT in high bodyweight group 


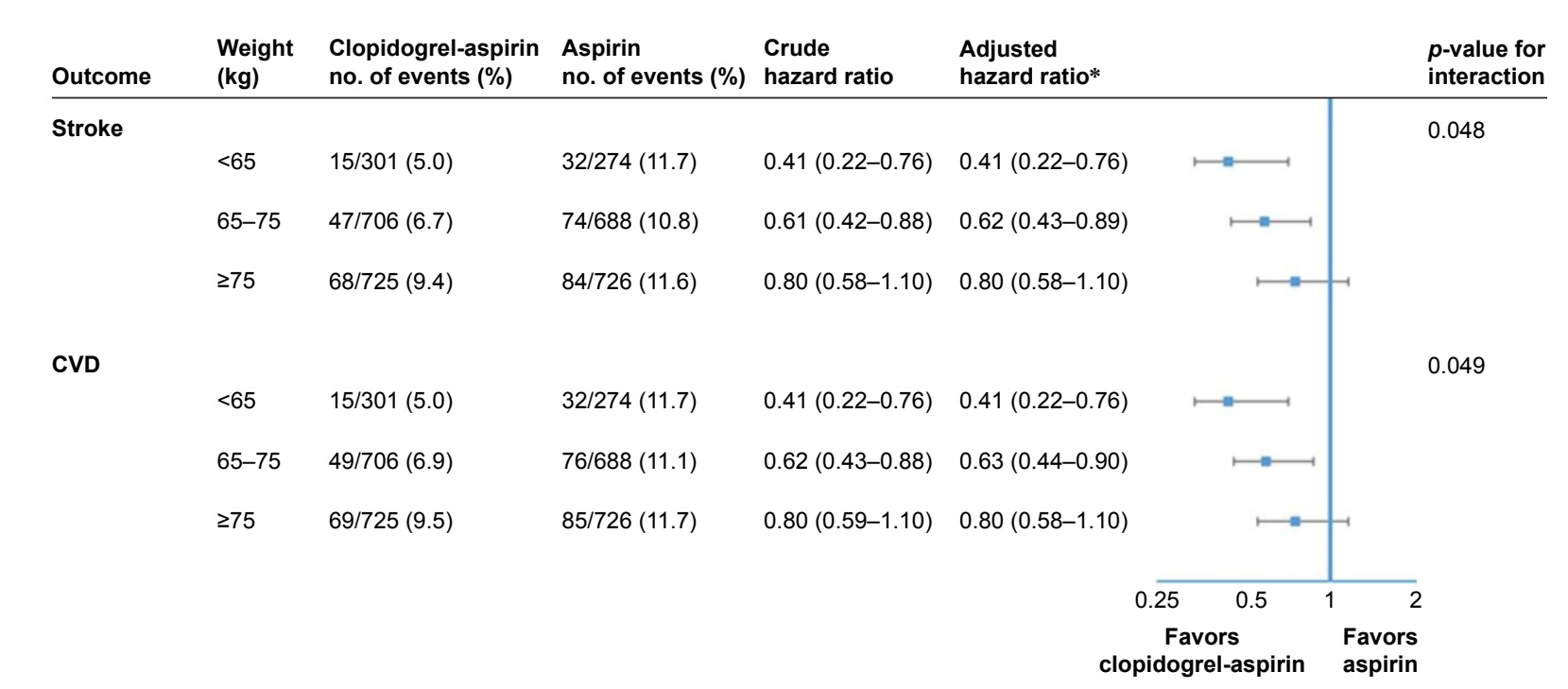

Figure 3 Hazard ratios for the primary and secondary end point.

Notes: Associations between antiplatelet treatment regime and efficacy outcomes in patients stratified by bodyweight. *Adjusted for age, gender, current or previous smoking, current or previous drinking, history of ischemic stroke or TIA, myocardial infarction, hypertension and hyperlipidaemia, final diagnosis of an acute minor ischemic stroke or TIA, the use of lipid lowering agents and antihypertension agents during 90-day follow-up period.

Abbreviations: CVD, cardiovascular disease; TIA, transient ischemic attack.

is the ceiling effect. Compared with some other pharmaceuticals in the market, antiplatelet agents are always prescribed in a uniform dosage, without conventional dose adjustments. From the practical standpoint, we should consider about dose modification for antiplatelet agents in different bodyweight patients. For the higher weight population, higher loading dose or triple therapy should be taken into account. Triple antiplatelet therapy vs DAT has been tested in several ongoing clinical trials. ${ }^{5,20}$ There are few trials focussing on the effect of bodyweight on antiplatelet therapy. This study provides clues for the research in this direction. Second, the incidence

Table 2 Safety outcomes between clopidogrel-aspirin and aspirin alone treatment in patients stratified by bodyweight

\begin{tabular}{llll}
\hline Safety outcome $^{\mathrm{a}}$ & $\begin{array}{l}\text { Weight } \\
\text { (kg) }\end{array}$ & $\begin{array}{l}\text { Clopidogrel-aspirin } \\
\text { no. of events (\%) }\end{array}$ & $\begin{array}{l}\text { Aspirin no. } \\
\text { of events (\%) }\end{array}$ \\
\hline Any bleeding & $<65$ & $\mathrm{I} / / 30 \mathrm{I}(3.7)$ & $6 / 274(2.2)$ \\
& $65-75$ & $9 / 706(\mathrm{I} .3)$ & $9 / 688(\mathrm{I} .3)$ \\
& $\geq 75$ & $\mathrm{I} 2 / 725(\mathrm{I} .7)$ & $9 / 726(\mathrm{I} .2)$ \\
Severe bleeding & $<65$ & $\mathrm{I} / 30 \mathrm{I}(0.3)$ & $\mathrm{I} / 274(0.4)$ \\
& $65-75$ & $0 / 706(0.0)$ & $2 / 688(0.3)$ \\
& $\geq 75$ & $\mathrm{I} / 725(0.1)$ & $\mathrm{I} / 726(0.1)$ \\
Moderate bleeding & $<65$ & $\mathrm{I} / 30 \mathrm{I}(0.3)$ & $0 / 274(0.0)$ \\
& $65-75$ & $0 / 706(0.0)$ & $\mathrm{I} / 688(0.2)$ \\
& $\geq 75$ & $\mathrm{I} / 725(0.1)$ & $\mathrm{I} / 726(0.1)$ \\
Mild bleeding & $<65$ & $4 / 30 \mathrm{I}(\mathrm{I} .3)$ & $3 / 274(\mathrm{I} .1)$ \\
& $65-75$ & $5 / 706(0.7)$ & $3 / 688(0.4)$ \\
& $\geq 75$ & $7 / 725(\mathrm{I} .0)$ & $3 / 726(0.4)$ \\
\hline
\end{tabular}

Note: asafety outcomes was based on the GUSTO definition.

Abbreviation: GUSTO, global utilization of streptokinase and tissue plasminogen activator for occluded coronary arteries. of clopidogrel resistance may be higher in patients with high bodyweight. Clinical trials have reported that high residual platelet activation after clopidogrel administration increases the risk of recurrent vascular events. ${ }^{21,22}$ The antiplatelet effect of $\mathrm{P} 2 \mathrm{Y}_{12}$-antagonists clopidogrel may depend on bodyweight in patients after ischemic stroke or TIA. In consideration of this potential mechanism, we may assess the effect of platelet function monitoring with treatment adjustment in overweight patients. And higher dose clopidogrel or novel P2 $\mathrm{Y}_{12}$-antagonists are needed to achieve sufficient platelet inhibition.

In present study, compared with MAT, DAT reduced the risk of stroke recurrence in low and middle bodyweight patients with minor stroke and TIA. The potential additional bleeding risk by DAT should also be assessed. The result showed that there was no significant difference in severe or mild bleeding among 3 groups. It indicates that DAT does not increase bleeding risk compared with MAT even in low bodyweight patients with minor stroke or TIA.

\section{Limitations}

This subgroup study has several limitations. First of all, the whole sample merely consisted of males, limiting broad applicability of findings. In addition, this was a subgroup analysis and may lack enough power to detect heterogeneities of treatment effects of DAT vs MAT among high weight patients. Furthermore, a large randomized and controlled clinical trial testing the effect of DAT on high weight population is needed to confirm this finding. 


\section{Conclusion}

In summary, to compare the effects of clopidogrel plus aspirin vs aspirin alone in secondary prevention of ischemic stroke or TIA patients with different bodyweight is rarely reported out of practical significance. And the result of this subgroup study showed that there were no statistically significant effects of clopidogrel plus aspirin vs aspirin single in those bodyweight $\geq 75 \mathrm{~kg}$. However, subgroup analyses can only generate hypotheses that should be tested in sufficiently powered RCTs. Therefore, our findings should be further validated in other populations because of this limitation.

\section{Acknowledgments}

The study was supported by grants from the Ministry of Science and Technology of the People's Republic of China (2016YFC1307300, 2013BAI09B03 and 2011BAI08B02), and a grant from Beijing Municipal Administration of Hospitals' Youth Programme (QML2015 0504).

\section{Disclosure}

The authors report no conflicts of interest in this work.

\section{References}

1. Wang Y, Wang Y, Zhao X, et al. Clopidogrel with aspirin in acute minor stroke or transient ischemic attack. N Engl J Med. 2013;369:11-19.

2. Wong KS, Wang Y, Leng X, et al. Early dual versus mono antiplatelet therapy for acute non-cardioembolic ischemic stroke or transient ischemic attack: an updated systematic review and meta-analysis. Circulation. 2013;128(15):1656-1666

3. Wong KS, Chen C, Fu J, et al; CLAIR study investigators. Clopidogrel plus aspirin versus aspirin alone for reducing embolisation in patients with acute symptomatic cerebral or carotid artery stenosis (CLAIR study): a randomised, open-label, blinded-endpoint trial. Lancet Neurol. 2010;9(5):489-497.

4. Kernan WN, Ovbiagele B, Black HR, et al. Guidelines for the prevention of stroke in patients with stroke and transient ischemic attack: a guideline for healthcare professionals from the American Heart Association/ American Stroke Association. Stroke. 2014;45(7):2160-2236.

5. Bath PM, Appleton JP, Beridze M, et al. Baseline characteristics of the 3096 patients recruited into the 'Triple Antiplatelets for Reducing Dependency after Ischemic Stroke' trial. Int J Stroke. 2017;12(5):524-538.

6. Bhatt DL, Fox KA, Hacke W, et al; CHARISMA Investigators. Clopidogrel and aspirin versus aspirin alone for the prevention of atherothrombotic events. $N$ Engl J Med. 2006;354(16): 1706-1717.
7. Kennedy J, Hill MD, Ryckborst KJ, Eliasziw M, Demchuk AM, Buchan AM; FASTER Investigators. Fast assessment of stroke and transient ischaemic attack to prevent early recurrence (FASTER): a randomised controlled pilot trial. Lancet Neurol. 2007;6(11):961-969.

8. Ng M, Fleming T, Robinson M, et al. Global, regional, and national prevalence of overweight and obesity in children and adults during 1980-2013: a systematic analysis for the Global Burden of Disease Study 2013. Lancet. 2014;384(9945):766-781.

9. Serena J, Segura T, Roquer J, Garcia-Gil M, Castillo J, Study A. The ARTICO study: identification of patients at high risk of vascular recurrence after a first non-cardioembolic stroke. BMC Neurol. 2015;15:28

10. Wang Y, Johnston SC, CHANCE Investigators. Rationale and design of a randomized, double-blind trial comparing the effects of a 3-month clopidogrel-aspirin regimen versus aspirin alone for the treatment of high-risk patients with acute nondisabling cerebrovascular event. Am Heart J. 2010;160(3):380-386. e381.

11. Harris RZ, Benet LZ, Schwartz JB. Gender effects in pharmacokinetics and pharmacodynamics. Drugs. 1995;50(2):222-239.

12. The Nutrition and Chronic Desease Status of Chinese People (2015) [online]. Available from: http://www.nhfpc.gov.cn. Accessed November 01, 2016

13. An international randomized trial comparing four thrombolytic strategies for acute myocardial infarction. The GUSTO investigators. $N$ Engl J Med. 1993;329:673-682.

14. Johnston SC, Easton JD, Farrant M, et al. Platelet-oriented inhibition in new TIA and minor ischemic stroke (POINT) trial: rationale and design. Int J Stroke. 2013;8(6):479-483.

15. Pankert M, Quilici J, Loundou AD, et al. Impact of obesity and the metabolic syndrome on response to clopidogrel or prasugrel and bleeding risk in patients treated after coronary stenting. Am J Cardiol. 2014;113(1):54-59.

16. Haberka M, Mizia-Stec K, Lasota B, Kyrcz-Krzemien S, Gasior Z Obesity and antiplatelet effects of acetylsalicylic acid and clopidogrel in patients with stable angina pectoris after percutaneous coronary intervention. Pol Arch Med Wewn. 2015;125(9):620-630.

17. Garcia-Labbe D, Ruka E, Bertrand OF, Voisine P, Costerousse O, Poirier P. Obesity and coronary artery disease: evaluation and treatment Can J Cardiol. 2015;31(2):184-194.

18. Badimon L, Hernandez Vera R, Padro T, Vilahur G. Antithrombotic therapy in obesity. Thromb Haemost. 2013;110(4):681-688.

19. Serebruany VL, Fortmann SD, Kim MH. Should the dose of antiplatelet drugs be adjusted for body weight? The example of vorapaxar. Cardiology. 2016;133(2):69-72.

20. TARDIS Trial Investigators, Krishnan K, Beridze M, et al. Safety and efficacy of intensive vs. guideline antiplatelet therapy in high-risk patients with recent ischemic stroke or transient ischemic attack: rationale and design of the Triple Antiplatelets for Reducing Dependency after Ischaemic Stroke (TARDIS) trial (ISRCTN47823388). Int $J$ Stroke. 2015;10(7):1159-1165.

21. Sibbing D, von Beckerath O, Schomig A, Kastrati A, von Beckerath N Impact of body mass index on platelet aggregation after administration of a high loading dose of $600 \mathrm{mg}$ of clopidogrel before percutaneous coronary intervention. Am J Cardiol. 2007;100(2):203-205.

22. Cuisset T, Frere C, Quilici J, et al. Relationship between aspirin and clopidogrel responses in acute coronary syndrome and clinical predictors of non response. Thromb Res. 2009;123(4):597-603. 


\section{Supplementary material Co-investigators of CHANCE trial}

Yongjun Wang, MD (Tiantan Hospital, Capital Medical University, principal investigator); Claiborne Johnston, MD, $\mathrm{PhD}$ (UCSF, principal investigator); Lawrence Wong, MD, $\mathrm{PhD}$ (Chinese University of Hong Kong, Prince of Wales Hospital, steering committee); David Wang, DO, FAHA, FAAN (University of Illinois College of Medicine, steering committee); James Wang, MD, PhD (University of Tennessee Health Science Center, steering committee); Mai N Nguyen-Huynh, MD, MAS (UCSF, steering committee); Chen Wang, MD (Tiantan Hospital, Capital Medical University, steering committee); Liying Cui, MD, PhD (Peking Union Medical College Hospital, steering committee); Yansheng Li, MD, PhD (Renji Hospital of Shanghai, steering committee); Qiang Dong, MD, PhD (Huashan Hospital of Fudan University, steering committee); Jianfeng $\mathrm{Xu}, \mathrm{MD}, \mathrm{PhD}$ (School of life sciences, Fudan University, steering committee); Jianping Jia, MD, PhD (Xuan Wu Hospital, Capital Medical University, steering committee); Jiang $\mathrm{Wu}, \mathrm{MD}, \mathrm{PhD}$ (First Hospital of Jilin University, steering committee); Jinsheng Zeng, MD, PhD (First Affiliated Hospital of Sun Yat-Sen University, steering committee); Xingquan Zhao, MD, PhD (Tiantan Hospital, Capital Medical University, steering committee); Liping Liu, MD, $\mathrm{PhD}$ (Tiantan Hospital, Capital Medical University, steering committee); Chunxue Wang, MD, PhD (Tiantan Hospital, Capital Medical University, steering committee); Yilong Wang, MD, PhD (Tiantan Hospital, Capital Medical University, steering committee); Zhimin Wang, MD (Taizhou First People's Hospital, site investigator); Yongjun Wang, MD (BeijingTiantan Hospital, site investigator); Haiqin Xia, MD (Taiyuan Iron And Steel Co., Ltd., General Hospital, site investigator); Bin Li, MD (Dagang Oilfield Gengeal Hospital, site investigator); Guiru Zhang, MD (Penglai People's Hospital, site investigator); Xudong Ren, MD (The Third People's Hospital of Datong, site investigator); Chunling Ji, MD (The Fourth Central Hospital of Tianjin, site investigator); Guohua Zhang, MD (The Second Hospital of Hebei Medical University, site investigator); Jianhua Li, MD (The First Hospital of Fangshan District, Beijing, site investigator); Bohua Lu, MD (Beijing Puren Hospital, site investigator); Liping Wang, MD (Tianjin Ninghe District Hospital, site investigator); Shutao Feng, MD (The People's Hospital of Zhengzhou, site investigator); Dali Wang, MD (Affiliated Hospital of North China Coal Medical College, site investigator); WeiguoTang, MD (Zhejiang Zhoushan Hospital, site investigator); Juntao Li, MD (Han Dan Central
Hospital, site investigator); Zhecheng People's Hospital Hongtian Zhang; Shanxi Medical University Second Hospital Guanglai Li; Baojun Wang, MD (Baotou Central Hospital, site investigator); Yuhua Chen, MD (The General Hospital of Changjiang River Shipping, site investigator); Ying Lian, MD (Dalian Economic And Technological Development Zone Hospital, site investigator); Bin Liu, MD (First Neurology Department, Affiliated Hospital of North China Coal Medical College, site investigator); Junfang Teng, MD (The First Affiliated Hospital of Zhengzhou University, site investigator); Rubo Sui, MD (First Affiliated Hospital of Liaoning Medical, site investigator); Lejun Li, MD (Lianyungang Municipal Hospital of Tcm, site investigator); Zhiling Yuan, MD (Central Hospital In Qiu County, site investigator); Dawei Zang, MD (Tianjin First Center Hospital, site investigator); Zuneng Lu, MD (Renmin Hospital of Wuhan University, site investigator); Li Sun, MD (Qingdao Central Hospital, site investigator); Dong Wang, MD (Baogang Hospital, site investigator); Liying Hou, MD (Changzhi City People's Hospital of Shanxi Province, site investigator); Dongcai Yuan, MD (HaLixun International Peace Hospital, site investigator); Yongliang Cao, MD (People's Hospital of Linzi District, Zibo, site investigator); Hui Li, MD (Yantai City Yantai Mountain Hospital, site investigator); Xiuge Tan, MD (Beijing Pinggu District Hospital, site investigator); Huicong Wang, MD (Taiyuan Central Hospital, site investigator); Haisong Du, MD (Chengde Central Hospital, site investigator); Mingyi Liu, MD (Shijiazhuang Central Hospital, site investigator); Suping Wang, MD (First Neurology Department, Dalian Municipal Central Hospital, site investigator); Qiuwu Liu, MD (Xian 141 Hospital, site investigator); Zhong Zhang, MD (Chengdu Third Municipal People's Hospital, site investigator); Qifu Cui, MD (Affiliated Hospital of Chifeng University, site investigator); Runqing Wang, MD (Zhengzhou Central Hospital, site investigator); Jialin Zhao, MD (Ningbo City, Zhejiang Province Lihuili Hospital Medical Center, site investigator); Jiewen Zhang, MD (Henan Provincial People's Hospital, site investigator); Jianping Zhao, MD (Jinzhong City Second Hospital, site investigator); Qi Bi, MD (Beijing Anzhen Hospital, Capital Medical University, site investigator); Xiyou Qi, MD (Beijing Huairou District Chinese Medicine Hospital, site investigator); Junyan Liu, MD (Hebei Medical University Third Hospital, site investigator); Changxin Li, MD (First Affiliated Hospital Shanxi Medical University, site investigator); Ling/Lv Li, MD (Hebei Provincial People's Hospital, site investigator); Xiaoping Pan, MD (Guangzhou First Municipal 
People's Hospital, site investigator); Junling Zhang, MD (Central Hospital In Cangzhou, site investigator); Derang Jiao, MD (The Chinese People's Armed Police Force Medical School Affiliated Hospital, site investigator); Zhao Han, MD (Zhejiang Wenzhou Medical College First Affiliated Hospital, site investigator); Dawei Qian, MD (Jilin Central Hospital, site investigator); Jin Xiao, MD (Anhui Maanshan Central Hospital, site investigator); Yan Xing, MD (Beijing Aviation Industry Central Hospital, site investigator); Huishan Du, MD (Luhe Hospital, Tongzhou District, Beijing, site investigator); Guang Huang, MD (Beijing Fuxing Hospital, Capital Medical University, site investigator); Yongqiang Cui, MD (The 306th Hospital of P.L.A, site investigator); Yan Li, MD (The First Affiliated Hospital of Tianjin University of Chinese Medicine, site investigator); Lianyuan Feng, MD (Baiqiuen International Peace Hospital of People's Liberation Army, site investigator); Lianbo Gao, MD (Fourth Affiliated Hospital of China Medical University, site investigator); Bo Xiao, MD (Xiangya Hospital Central-South University, site investigator); Yibin Cao, MD (Tangshan Worker's Hospital, site investigator); Yiping Wu, MD (The 1st Hospital In Handan, site investigator); Jinfeng Liu, MD (Yangquan Coal (Group) Co., Ltd. General Hospital, site investigator); Zhiming Zhang, MD (Tianjin Tianhe Hospital, site investigator); Zhengxie Dong, MD (Nantong First People's Hospital, site investigator); Limin Wang, MD (The 1st Hospital of Zhangjiakou City, site investigator); Li He, MD (West China Hospital, Sichuan University, site investigator); Xinchen Wang, MD (The Second Affiliated Hospital of Shandong University of TCM, site investigator); Xueying Guo, MD (Fenyang Hospital of Shanxi Province, site investigator); Ming Wang, MD (Zhejiang Zhoushan Putuo District People's Hospital, site investigator); Xiaosha Wang, MD (Xiyuan Hospital of China Academy of Chinese Traditional Medicine, site investigator); Jiandong Jiang, MD (No 2 People's Hospital East In Lianyungang City, site investigator); Renliang Zhao, MD (Affiliated Hospital of Qingdao University Medical College, site investigator); Shengnian Zhou, MD (Qilu Hospital of Shandong University, site investigator); HaoHu, MD (Zibo Hospital of Traditional Chinese Medicine, site investigator); Maolin He, MD (Beijing Shijitan Hospital, site investigator); Fengchun Yu, MD (Beijing Haidian Hospital, site investigator); Quping Ouyang, MD (Beijing Shunyi District Hospital, site investigator); Jingbo Zhang, MD (Dalian Third Municipal Hospital, site investigator); Anding Xu, MD (The First Affiliated Hospital of Jinan University, site investigator); Xiaokun Qi,
MD (Navy Genaral Hospital of P.L.A, site investigator); Lei Wang, MD (Beijing Second Artillery General Hospital, site investigator); Fuming Shi, MD (Beijing Daxing District Hospital, site investigator); Fuqiang Guo, MD (Sichuan Province People's Hospital, site investigator); Jianfeng Wang, MD (Dalian Municipal Central Hospital, site investigator); Fengli Zhao, MD (The Second Hospital In Baoding, site investigator); Ronghua Dou, MD (The Hospital Combine Traditional Chinese And Western Medicine In Cang zhou, site investigator); Dongning Wei, MD (The 309th Hospital of PLA, site investigator); Qingwei Meng, MD (Liangxiang Hospital of Fangshan District, Beijing, site investigator); Yilu Xia, MD (HuaXin Hospital First Hospital of Tsinghua University, site investigator); Shimin Wang, MD (TianjinHuanhu Hospital, site investigator); Zhangcang Xue, MD (Shijiazhuang First Hospital, site investigator); Yuming Xu, MD (The First Affiliated Hospital of Zhengzhou University, site investigator); Liping Ma, MD (Xinzhou City People's Hospital, site investigator); Chun Wang, MD (Sichuan Province People's Hospital of Deyang City, site investigator); Jiang Wu, MD (First Hospital, Jilin University, site investigator); Yifeng Du, MD (Shandong Provincial Hospital, site investigator); Yinzhou Wang, MD (Fujian Province Hospital, site investigator); Lijun Xiao, MD (Liaoyang City Third People's Hospital, site investigator); Fucong Song, MD (Handan City Center Hospital, site investigator); Wenli Hu, MD (Beijing Chaoyang Hospital, Capital Medical University, site investigator); Zhigang Chen, MD (Beijing University of Chinese Medicine East Hospital, site investigator); Qingrui Liu, MD (Hebei Medical University Fourth Hospital, site investigator); Jiemin Zhang, MD (The Fourth Affiliated Hospital of Soochow University, site investigator); Mei Chen, MD (Zhejiang University of Chinese Medicine Hospital Affiliated First, site investigator); Xiaodong Yuan, MD (Affiliated Hospital of Kailuan Company Ltd, site investigator); Zhihui Liu, MD (Affiliated Hospital of Weifang Medical University, site investigator); Guozhong Li, MD (The First Hospital of Harbin Medical University, site investigator); Xiaohong Li, MD (Dalian Friendship Hospital, site investigator); Tingchen Tian, MD (Tianjin Dagang Hospital, site investigator); Xia Meng, MD, PhD (Tiantan Hospital, Capital Medical University, member of coordinating center); Jinxi Lin, MD, PhD (Tiantan Hospital, Capital Medical University, member of coordinating center); Anxin Wang, MD (Tiantan Hospital, Capital Medical University, member of coordinating center); Yong Zhou, PhD (Tiantan Hospital, Capital Medical University, member of coordinating center); Xianwei Wang, MD 
(Tiantan Hospital, Capital Medical University, member of coordinating center); Chao Li, MD (Tiantan Hospital, Capital Medical University, member of coordinating center); Yuan Shen, MD (Tiantan Hospital, Capital Medical University, member of coordinating center); Xianhong
Liang, MD (Tiantan Hospital, Capital Medical University, member of coordinating center); Jing Jing, MD, PhD (Tiantan Hospital, Capital Medical University, member of coordinating center).

\section{Publish your work in this journal}

Therapeutics and Clinical Risk Management is an international, peerreviewed journal of clinical therapeutics and risk management, focusing on concise rapid reporting of clinical studies in all therapeutic areas, outcomes, safety, and programs for the effective, safe, and sustained use of medicines. This journal is indexed on PubMed Central, CAS,
EMBase, Scopus and the Elsevier Bibliographic databases. The manuscript management system is completely online and includes a very quick and fair peer-review system, which is all easy to use. Visit http://www.dovepress.com/testimonials.php to read real quotes from published authors. 